\title{
COMPUTER TOOLS IN THE TEACHING OF TRANSLATORS AS WEBSITE LOCALIZATION PROFESSIONALS
}

\author{
J. Gutiérrez-Artacho, M.D. Olvera-Lobo \\ University of Granada (SPAIN)
}

\begin{abstract}
Website localization constitutes a new field of study and professional intervention. Localization can be described as a global process in which globalization and internationalization come together in an effort to prepare a particular product for a particular locale. We could define locale as the collection of features of the user's environment that is dependent on language, country/region, and cultural conventions. Locales usually provide more information about cultural conventions than about languages. As a result, the term localization remains irremediably linked to the concept of culture. Correct localization cannot be achieved without knowing and bearing in mind the locale to which it is directed.

It is also necessary to point out that localization is a modality of translation. Localization involves translation of textual content into language and textual conventions of the target locale and adaptation of non-textual content as well as input, output and delivery mechanisms to take into account the cultural, technical and regulatory requirements of that locale. In sum, localization is not so much about specific tasks as it is about the processes by which products are adapted.

The translation process requires language professionals to perform various roles, completing each stage on time and undertaking multiple tasks in order to meet the conditions for delivery of the translation in accordance with a series of quality parameters [1-10]. For its part, localization involves a variety of agents in the localization process, though their number may vary from a single person responsible for the entire process to a multiplicity of agents in large organizations, including business managers, localization managers, localization engineers, terminologists, locators, quality control operators and freelance translators, among others. The process also varies considerably depending on the nature of the project, the technologies involved, the resources available and even the type of translation process employed.
\end{abstract}

The particularities of this translation modality make the use of translation tools, also known by the acronym CAT (Computer Assisted Translation) tools, a necessity. Furthermore, we should not forget that these particularities complicate the translation process in terms of the time and effort required. In order to raise the students of the Bachelor's Degree in Translation and Interpretation to the level required by this hyper-competitive market, production times must be reduced, and this is where CAT tools come into their own. While professional website translators-localisers form part of a complex team, they often do not participate in the decisions taken in the primary stages of web localization. With this in mind we must forget the concept of the localiser as an isolated element and begin to see them as an integral part of a complex production chain.

With the right training a web content translator may be qualified to carry out several of these functions. In any case they would always have a wide range of CAT tools available to make the job easier. Bearing this in mind, the primary objective of our research $t$ is to define and analyse CAT tools that are useful for training web content translators-localisers at any stage of the translation project. We have done a classification of the best Localization tools according to the different stage of the process.

Keywords: Website localization, translation, computer tools, CAT Tools, University.

\section{INTRODUCTION}

The European Higher Education Area (EHEA) supposes a change in the organization of teaching and learning methods, focused on skills training. The Tuning Project, developed by the European Commission, has been an important basis for transformations in the university curricular reform within the European convergence for higher education. This project classifies skills into generic (transversal) and specific (professional) types. This implies, as well as the achievement of the objectives established in each study plan subject along with their specific competencies, the acquisition of competencies and skills of a more general -and transversal-, nature. In effect, the activities 
developed in the globalised sphere of the current labour market are dynamic and demand, along with specialised skills and knowledge, certain attitudes, aptitudes and personality traits, many of which can be strengthened via the acquisition of so-called transversal skills.

In the Spanish university system, the training of translators at an undergraduate level generally combines two social constructivist teaching and learning approaches. The first and most widely used of these is project based learning (PBL) [5], exemplified in Translation Studies literature through different works [6-13]. The second is cooperative learning (CL) [14], which is perhaps less applied and closely linked to collaborative learning (CoL) [15]. Teaching proposals such as PATT (Professional Approach to Translator Training) [7,11] combine these two well established approaches for learning in the classroom, namely, project-based and cooperative/collaborative learning.

The ever-demanding translation labour market has recently become, above all, a market dominated by the so-called localization processes. Thus, we talk about localization of software, apps, videogames and websites, each with its own singularities [16]. The acronym GILT (Globalization, Internationalization, Localization and Translation) is used to define the group of tasks and strategies aimed at creating a digital global product. In this manner, where translation focuses on reproducing a text in a source language to a target destination, localization (which can be considered as a mode of translation) is a global process with the aim of adapting a product or service to a determined language, culture or local aspect, that is, a locale. In this context, the term internationalization refers to a specific pre-localization process that implies the inclusion of technical elements in the original product to minimise the subsequent need for redesign or reengineering. It also deals with the cultural implications of original content, such as the use of colors, images and icons, amongst other characteristics. Finally, internationalization and localization form part of the globalization cycle, which is situated in a general plan and refers to the transfer of goods, services, people, technology and information in a universal and multicultural environment. That is, globalization is responsible for making commercialization possible and, therefore, enabling the computer product or website to reach any market regardless of language or culture.

It is because of this that GILT places interlinguistic communication on the wider globalization stage, and also underlines the specific processes necessary for dealing with electronic content. Knowledge of these concepts means that translation professionals are aware of their role in this enormous sphere of globalization. So, translators, as interlinguistic communication professionals, play an important role and constitute an active part of globalization. The different stages of the localization process are, in short: (i) order receipt and processing, (ii) organization of human resources, (iii) organization of material resources, (iv) translation or localization (v) quality control (vi) content inclusion, (vii) assessment and, lastly (viii) delivery [17].

GILT practices continue to be developed in response to market demands, whilst academic studies and their teaching application in this dynamic domain are still falling behind, with industry leading the way. Likewise, as mentioned above, client demands have given rise to a number of localization subspecialisations such as web localization. Specifically referring to the latter, we draw attention to the fact that the website translator forms part of a complex team, and decision-making during the first stages of web localization do not require his or her participation [17], which can result in deficiencies in the quality of the final product. The concept of localizer as an isolated element should, then, be abandoned and replaced by a perception of this person as part of a complex production chain. The number of links in the localization process for a web resource will depend to a large extent on the size of the company and the available human resources [17]. In general terms, the members of a web localization project are (i) the management, (ii) the commercial and administrative manager, (iii) the project manager, (iv) the engineers or IT specialists, (v) in-house translators and (vi) external translators [18].

The particularities of this modality of translation involve working with diverse files and formats (image, audio, video). It should not be forgotten that, for these types of assignments, the technical and cultural specifications complicate the translation process in relation to the time and effort required. Thus, a reduction in production times is needed to respond to the hyper-competitiveness of the market, without sacrificing quality in the final result. Computer assisted translation (CAT) tools, are very useful for achieving this objective.

Even with the awareness of the speed at which market demands are evolving, and the training needs of future professionals, appealing to the responsibility of academic institutions to respond to these changes is indisputable. With the correct training, a web content translator would be qualified to carry 
out a number of the functions associated with the aforementioned localization tasks [17]. The objective of CAT tools is to facilitate these.

With the aim of determining the most widely-used tools useful for localization amongst translation researchers and professionals (especially web localization), we analysed related information collected in two of the most relevant bibliographical databases (SCOPUS and Web of Science), and the very popular Google Scholar. We began from the premise that specialised publications collect updated information from the different spheres of knowledge. With the purpose of carrying out a bibliographical revision of the sources, the searches focused on web localization and on the tools involved at each stage of this process.

Below we set out the study carried out in detail, which allowed us to establish the CAT tools with the most widespread use at the different stages of the localization process. We consider, however, that this simple methodology could be encouraging for teachers to adopt their academic programmes to the professional reality of these university students.

\section{COMPUTER ASSISTED TRANSLATION: LOCALIZATION TOOLS}

A qualitative methodology was followed for this study. We put queries to the Scopus and Web of Science databases, and the Google Scholar web tool. Although we considered the particularities of the consultation interfaces for these tools, we tried to use, as far as possible, equivalent search parameters in order to obtain results that were comparable. The searches were carried out in November 2017.

Scopus is a database with a wide subject range, covering around $80 \%$ of expert-reviewed international scientific publications. In addition, its contents are updated on a weekly basis. To carry out the consultation, we used the following equation in the advanced search option:

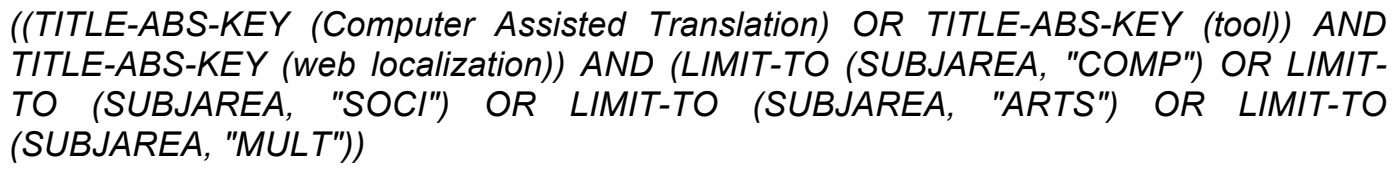

The term CAT was searched in the title, abstract and keyword fields (TITLE-ABS-KEY), along with the terms tool and web localization, all under the same TITLE-ABS-KEY tag. Lastly, the subject areas Social Sciences (SUBJAREA, "SOCl"), Arts and Humanities (SUBJAREA, "ARTS") and Multidisciplinary (SUBJAREA, "MULT") were selected via the (LIMIT-TO) delimiter.

Web of Science is a database that, like Scopus, boasts a wide subject variety. It contains publications from the world's most prestigious magazines and publishers on all areas of the sciences, social sciences, art and humanities. We formulated the following search equation via the advanced search function:

\section{((TS=("Computer assisted translation") OR (TS=(tool)) AND (TS=("web localization") AND Refined by: RESEARCH DOMAINS: (ARTS HUMANITIES) AND RESEARCH AREAS: (LITERATURE OR LINGUISTICS OR ARTS HUMANITIES OTHER TOPICS OR $A R T)$}

The term Computer Assisted Translation was searched for in the topic search (TS) field, along with tool and web localization.

Google Scholar is a product which, in contrast to traditional databases, does not execute a transfer of magazine content; rather it systematically trawls the web. To do so it follows the same philosophy as Google, but with a convergence of two services on a single platform. On the one hand, it is a scientific publication search engine and, on the other, it is a citation index (as is Web of Science), which aids in finding out the impact of publications. The search equation we formulated was as follows:

tool OR CAT OR "Computer Assisted Tool" "web localization"

With the aim of discovering the tools mentioned in these specialized works we proceeding to the analyse the content of the results recovered in each database. The highest number of publications was found on Scopus (181) followed by Google Scholar (97) and, lastly, Web of Science (43). 


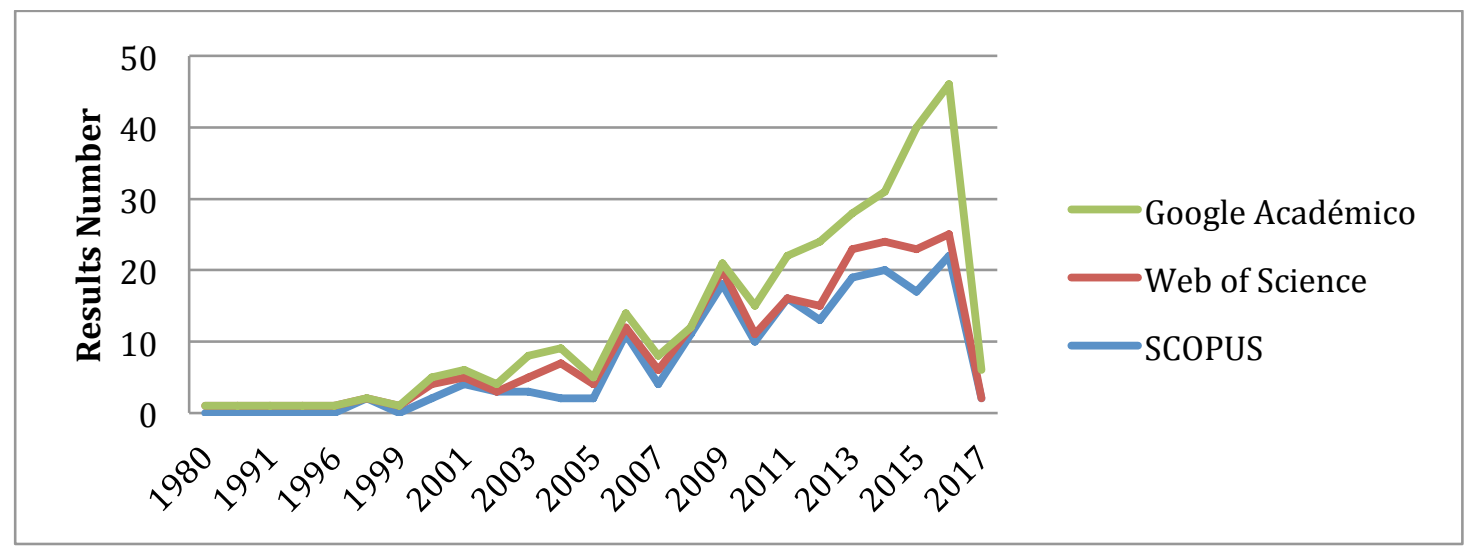

Figure 1: Results/year of publication on Google Scholar, SCOPUS and Web of Science

However, following a detailed revision of the complete text, we were able to confirm the scarce existence of publications referring to localization tools dealing exclusively with translation as the main research subject. The majority of the articles are published in magazines whose central topic is computational sciences, as well as IT in general. Of the publications that were particularly relevant for our topic of analysis, we identified a large group of tools implicated in localization processes. We then established a classification of these, organizing them around eight large types (Table 1).

Table 1. Computer tools for web localization

\begin{tabular}{|l|c|l|}
\hline & $\begin{array}{l}\text { Total no. } \\
\text { of tools }\end{array}$ & Programs \\
\hline Assisted translation & 23 & $\begin{array}{l}\text { Across Personal Edition, Anaphraseus, Araya, Atlas, CafeTran, } \\
\text { DéjàVu, EsperantiloTM, MemoQ, Memsource, MetaTexis, } \\
\text { MultiTrans, OmegaT, OpenTM2, SDL Trados Studio, Swordfish, Tr- } \\
\text { AID, Wordfast }\end{array}$ \\
\hline $\begin{array}{l}\text { Specific tools for web } \\
\text { localization }\end{array}$ & 9 & $\begin{array}{l}\text { Alchemy Catalyst, CatsCradle, Drupal Localization System, } \\
\text { Globalsight, Localized Website Design Advisor, Lokalize, Poedit, } \\
\text { SDL Passolo, Virtaal }\end{array}$ \\
\hline Automatic translation & 6 & Crossroad, Dr.Eye, LabelTranslator, LogoVista, PROMT, TransWhiz \\
\hline Translation memories & 2 & MEMOrg, MemoQ \\
\hline Terminology managers & 5 & SDL MultiTerm, TermExt, TermStar, WordSmith Tools \\
\hline Text and corpus analysis tool & 3 & AntConc, TextStat, Translearn \\
\hline Web accessibility & 3 & Acrolinx, aDesigner, Clarifai \\
\hline Other tools & 3 & Alipi.us, AppleTrans, JILT - Bookmarklet \\
\hline
\end{tabular}

CAT tools: They are translation tools that include the use of software to help people translate. In cases where there are time constraints, a computer assisted translation tool can effectively reduce translation time, allowing the translator to translate content and meet deadlines. SDL Trados is currently the most widely used on the market.

Website localization tools: Normally consists of tools that facilitate the translation of the user interface, online help and the documentation that accompanies the product. They tend to be extremely varied and are used when computer assisted translation programs reject certain formats. These tools also include, on certain occasions, the possibility of web page testing, as they permit the introduction of bugs (errors in the application) if the source code has not been duly separated from the interface text.

Automatic translation: Automatic translation has produced good results for Western European languages, even with morphologically rich languages such as German, but it still faces innumerable challenges when the languages are very different (Spanish-Japanese) or where the casuistry is too diverse as a result of a large number of declensions and genders, etc. The text offered by an 
automatic translator generally requires post-edition. They can be useful above all in environments where limited lexicons and predetermined grammatical structures are employed.

Translation memories (TM): A translation memory is a type of program that suggests translations previously stored in the memory that show a determined percentage of correspondence regarding the new text segment to be processed. A translation memory is a linguistic database that continuously stores translations for future use.

Terminology managers: A terminology manager, also called a termbase manager, is a software program comprising an expandable database that allows the management -creation, management and extraction - of data by users. These tools provide support to a category model with an onomasiological orientation. Some of the main CAT programs offer additional termbase software, which is the case for SDL Multiterm.

Text and corpus analysis tool: Tools which integrate functions for lexical labelling and partial syntaxic analysis of source sentences. With these tools localizers can achieve complete analyses of the sentences in a way that it allows them to reduce the supervision time for a corpus.

Web accessibility: This group comprises the automatic accessibility review tools that permit you to check whether verification points are being complied with regarding accessibility guidelines, which can be assessed automatically.

Other tools: Finally, we included those tools that cannot be included in the previous sections but provide effective help for web localizers to carry out their work.

The knowledge of the evolution of the translation profession in its different aspects is essential for responding to the constant challenges it creates. In professions as closely linked to information and communication technologies as this, advances are dizzying, and it is essential to keep up-to-date in matters such as existing computer tools. The appropriateness of academic programmes for the different subjects that students must take for the changes that occur is an unavoidable obligation and responsibility.

\section{CONCLUSIONS}

The skills that a web localizer must attain are, to a certain extent, the same as those that a translator must have. Nevertheless, the new professional field referred to as localization is not simply a different type of translation activity. In contrast, it is a complex process of product adaptation and even, in many cases, of transcreation, which has distinguishing attributes. In effect, as we have already mentioned, localization involves [19]:

a) translation of textual content into language and textual conventions of the target locale; and

b) adaptation of nontextual content (from colors, icons and bitmaps, to packaging, form factors, etc.) as well as input, output and delivery mechanisms to take into account the cultural, technical and regulatory requirements of that locale.

For these professionals, adapting to this new work profile and environment is paramount. This study has enabled the identification of a number of the useful computer tools in the field of a web localizer, according to their presence on specialised publications. In addition, it offers a classification of these tools and, although it is not exhaustive either in terms of typology or products, it does refer to the tools mentioned by researchers and specialists. These tools do not just focus on text editing, they include other website-specific elements (such as images with alternative text), and other localization-specific stages (such as project or terminology management).

There are now, fortunately, numerous training initiatives originating in the academic world that endeavour to respond to the needs of the real world. In this regard, institutions have begun to adopt a greater commitment towards professional demands from industry, and from the information society. The objective of our proposal is to offer a modest contribution to this achievement.

\section{ACKNOWLEDGEMENTS}

The authors are grateful to the Spanish "Ministry of Science and Innovation" for supporting this study, in the framework of the research Project CSO2015-64532-R, partially funded by the FEDER program of the European Union. 


\section{REFERENCES}

[1] M.D. Olvera-Lobo, B.J. Robinson, M.R. Castro-Prieto, E. Quero-Gervilla, R.Muñoz-Martín, E. Muñoz-Raya, M. Murillo-Melero, J.A. Senso-Ruiz, B. Vargas-Quesada, J.L.Díez-Lerma, "A professional approach to translator training (PATT)“. Meta: Journal des Traducteurs, vol. 52, no. 3, pp. 517-528, 2007.

[2] M.D. Olvera-Lobo, B.J. Robinson, J.A. Senso-Ruiz, R. Muñoz-Martín, E. Muñoz-Raya, M. Murillo-Melero, E. Quero-Gervilla, M.R. Castro-Prieto, T. Conde-Ruano, "Student satisfaction with a Web-based collaborative work platform“. Perspectives: Studies in Translatology, vol. 15, no. 2, pp. 106-122, 2008.

[3] M.D. Olvera-Lobo, B.J. Robinson, J.A. Senso-Ruiz, R. Muñoz-Martín, E. Muñoz-Raya, M. Murillo-Melero, E. Quero-Gervilla, M.R. Castro-Prieto, T. Conde-Ruano, "Teleworking and collaborative work environments in translation training". Babel: International Journal of Translation, vol. 55, no. 2, pp. 165-180, 2009.

[4] B.J. Robinson, M.D. Olvera-Lobo, J. Gutiérrez-Artacho, "After Bologna: Learner- and competence-centred translator training for "digital natives"', in From the Lab to the Classroom and Back Again: Perspectives on Translation and Interpreting Training. New Trends in Translation Studies Series (Martín de León, C.; González-Ruiz, V. (eds.). Frankfurt am Main: Peter Lang, pp. 325-359, ISBN: 978-3-0353-9553-2, DOI: 10.3726/978-3-0353-0806-8, 2016.

[5] R. Ribé, N. Vidal, Project Work. Step by step. Heinemann, Oxford, 1993.

[6] M.D. Olvera Lobo, et al. "Translator Training and Modern Market Demands". Perspectives: Studies in Translatology, vol. 13, no. 20, pp. 132-142, 2005.

[7] M.D. Olvera-Lobo, "A professional approach to translator training (PATT)". Meta, Journal des traducteurs, vol. 52n no. 4, pp. 517-528, 2007.

[8] J. Gutiérrez-Artacho, M.D. Olvera-Lobo, C.I. Hunt-Gómez, "Incorporación de las nuevas tecnologías de la innovación y un modelo didáctico adaptado a la formación en traducción e interpretación“. III Congreso Internacional sobre Innovación Pedagógica y Praxis Educativa. Libro de Actas, 28-30 de noviembre de 2016, Sevilla (España). Sevilla: AFOE Formación, pp. 1030-1039, ISBN: 978-84-608-8348-7, 2017.

[9] Olvera-Lobo, M.D. et al. "Collaborative Work Learning in Higher Education". In Encyclopedia of Networked and Virtual Organizations (Putnik, G. D. and Cunha, M.M. (ed.). Idea Group, Hershey, pp. 261-268, 2008.

[10] J. Gutiérrez-Artacho, M.D. Olvera-Lobo, M.D. "Gamification in the Translation and Interpreting degree: A new methodological perspective in the classroom". EDULEARN16 Proceedings: 8th International Conference on Education and New Learning Technologies (Gómez Chova, L.; López Martínez, A.; Candel Torres, I. (eds.), 4-6 july, Barcelona (España). Valencia: IATED Academy, pp. 50-58, ISBN: 978-84-608-8860-4, DOI: 10.21125/edulearn.2016.1008, 2016.

[11] B.J. Robinson, M.D. Olvera-Lobo, J. Gutiérrez-Artacho. "After Bologna: Learner- and competence-centred translator training for 'Digital natives'. In From the lab to the classroom and back again: Perspectives on translation and interpreting training (C. Martín de León, and V. González-Ruiz (eds.), Peter Lang, Frankfurt am Main, pp. 325-359, 2016.

[12] D.C. Kiraly, "Project-based Learning: A Case for Situated Translation". Meta, vol. 50, no. 4, pp. 1098-1111, 2005.

[13] I. Durán Muñoz, "El trabajo en equipo y las TICs en la enseñanza de traducción especializada". In TIC, trabajo colaborativo e interacción en terminología y traducción (C. Vargas Sierra (ed.), Comares, Granada, pp. 71-78, 2014.

[14] D.W. Johnson, R.T. Johnson, E.J. Holubec, Circles of Learning: Cooperation in the Classroom. Interaction Book Company, Edina, Minnesota, 1986.

[15] E.F. Barkley, K.P. Cross, C.H. Major, Collaborative Learning Techniques: A Handbook for College Faculty (2nd ed.). Jossey-Bass, San Francisco, 2014.

[16] P. Sandrini, "Localization and Translation". MuTra Journal, no. 2, 2008. 
[17] A. Estellés, "Revisión del entorno de trabajo de un traductor de contenido web". Tradumàtica, vol 8, pp. 0001-12, 2010.

[18] J. Parra, "El mercado laboral en el sector de la localización". La traducción científico-técnica y la terminología en la sociedad de la información, pp. 207-214, 2002.

[19] K. Dunne, "A Copernican revolution". In: Perspectives on Localization (Keiran Dunne, ed.) Amsterdam: John Benjamins, pp. 1-11, 2006. 